Historic, Archive Document

Do not assume content reflects current scientific knowledge, policies, or practices. 



\begin{tabular}{|c|c|c|c|}
\hline SEEDS & $\begin{array}{l}\text { Weight } \\
\text { Per Bu. }\end{array}$ & $\begin{array}{l}\text { Pounds } \\
\text { Per Aere }\end{array}$ & TIME OF SEEDING \\
\hline $\begin{array}{l}\text { Alfalfa, } \\
\text { broadcast.... }\end{array}$ & 60 & $10-20$ & April-August \\
\hline Alsike, broadcast & 60 & $7-10$ & February-August \\
\hline Barley, drilled 8 & 48 & $72-120$ & March-May \\
\hline $\begin{array}{l}\text { Blue Grass, } \\
\text { broadcast.... }\end{array}$ & 14 & $15-20$ & Mar. Apr.-Jul. Aug. \\
\hline $\begin{array}{l}\text { Buckwheat, } \\
\text { drilled 8...... }\end{array}$ & 48 & $35-60$ & June--July \\
\hline $\begin{array}{l}\text { Clover, Crimson } \\
\text { broadcast. }\end{array}$ & 60 & 10-15 & July-August \\
\hline $\begin{array}{c}\text { Clover, Red, } \\
\text { broadcast }\end{array}$ & 60 & 8-12 & Feb. Apr.-August \\
\hline $\begin{array}{c}\text { Clover, Mammoth } \\
\text { broadcast................ }\end{array}$ & 60 & 8-12 & Feb. Apr.-August \\
\hline $\begin{array}{l}\text { Clover, Sweet, } \\
\text { hulled, broadcast }\end{array}$ & 60 & $10-20$ & March-August \\
\hline $\begin{array}{l}\text { Clover, White } \\
\text { broadcast. }\end{array}$ & 60 & $6-8$ & February-August \\
\hline $\begin{array}{l}\text { Fescue, Meadow } \\
\text { broadcast. }\end{array}$ & 12 & $15-20$ & Mar. Apr.-July Aug. \\
\hline Flax, drilled 8 & 56 & $28-56$ & May \\
\hline $\begin{array}{l}\text { Millet, German } \\
\text { drilled 8. }\end{array}$ & 50 & $25-50$ & May-June \\
\hline $\begin{array}{l}\text { Millet, Hungarian } \\
\text { drilled } 8 \ldots \ldots \ldots . . . . . .\end{array}$ & 48 & $25-50$ & May-July \\
\hline $\begin{array}{l}\text { Orchard Grass } \\
\text { broadcast....... }\end{array}$ & 14 & $15-25$ & Mar. Apr.-Jul. Aug. \\
\hline $\begin{array}{l}\text { Peas, Canada, } \\
\text { drilled } 8\end{array}$ & 60 & $120-180$ & March-April \\
\hline $\begin{array}{l}\text { Rape, Dwarf } \\
\text { Essex, broadcast }\end{array}$ & 50 & $5-8$ & April-July \\
\hline $\begin{array}{l}\text { Red Top, hulled, } \\
\text { broadcast...-.-. }\end{array}$ & 14 & $10-15$ & Mar. Apr.-Jul. Aug. \\
\hline $\begin{array}{l}\text { Rye, Winter, } \\
\text { drilled } 8\end{array}$ & 56 & $85-115$ & September--Nov. \\
\hline $\begin{array}{l}\text { Soy Beans, } \\
\text { drilled } 8 . .\end{array}$ & 60 & $60-120$ & May-June \\
\hline $\begin{array}{l}\text { Timothy, } \\
\text { broadcast.... }\end{array}$ & 45 & $10-15$ & Mar. July-Sept. \\
\hline $\begin{array}{l}\text { Vetch, Spring, } \\
\text { drilled 8 }\end{array}$ & & $30-60$ & April-May \\
\hline $\begin{array}{l}\text { Vetch, Winter, } \\
\text { drilled } 8\end{array}$ & 60 & $30-60$ & July-September \\
\hline $\begin{array}{l}\text { Wheat, Winter, } \\
\text { drilled } 8\end{array}$ & 60 & $75-135$ & September-Oct. \\
\hline $\begin{array}{l}\text { Wheat, Spring, } \\
\text { drilled } 8\end{array}$ & & $90-120$ & April \\
\hline
\end{tabular}

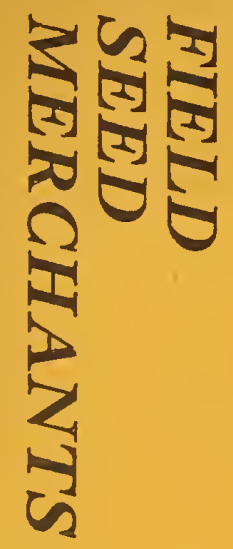

$$
\therefore
$$





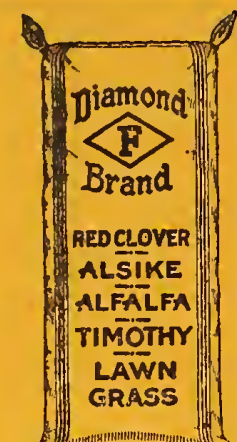

Next Issue, Aug. 5

TRRMS:-Caghto

No Digcount

Quotations for prompt shipment subject market changes and unsold. Prices f.o.b. Toledo.

W. FLOWER CO.

802 Lafayette St.

TOLEDO, OHIO

July 22, 1927

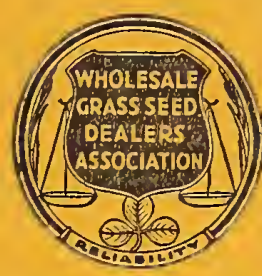

Telephone

MAin 6191

Telephone calls and telegrams cest money. When they come our asking more money for our gonds.

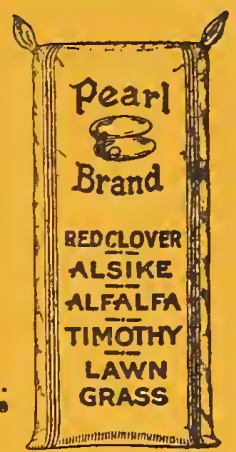

Bags extra at value unless olherwise specified, not returnable Printers and manifest errors excepled.

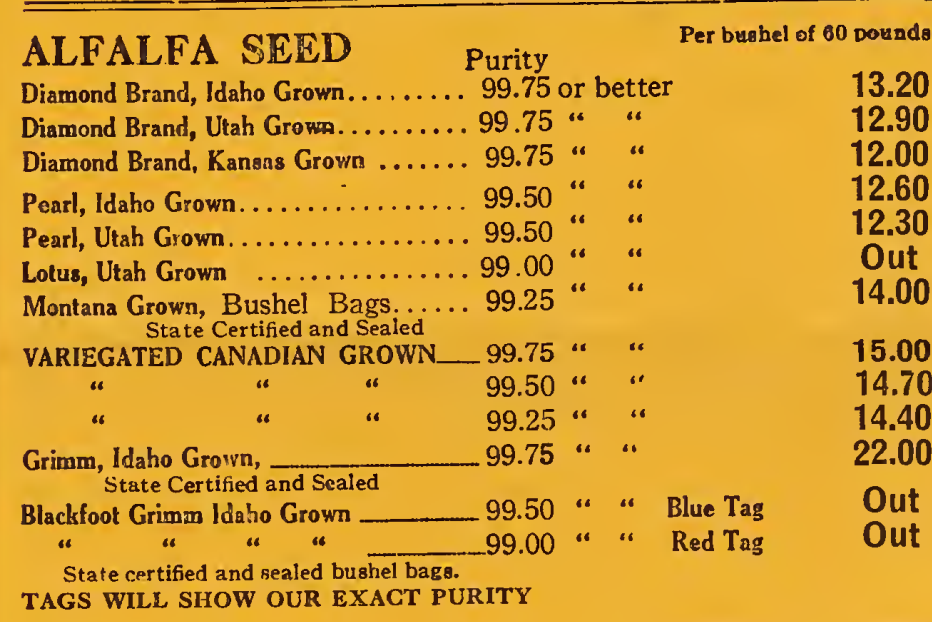

13.20

12.00

12.60

12.30

14.00

15.00

14.70

22.00

Out

\section{ALSIKE SEED}

DIAMOND BRAND

OUR PRIME

PEARL

LOTUS

PANSY

MIXED Alsike and Timothy

TAGS WILL SHOW OUR EXACT PUR1TY

\section{CLOVER SEED}

Medium - Domestic Grown
Purity 99.50 or better

99.00 “" “

98.50 " "

97.00 “

95.00 " "
Per buahel of 60 pounde

Prices

on

Appli-

cation
Out

$$
\text { Silv }
$$

\section{GRASS SEEDS}

BLUE GRASS (Gross for net sacks free)

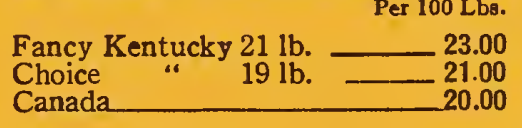

\begin{tabular}{l} 
Fancy Kentucky $21 \mathrm{lb}$. \\
Choice $19 \mathrm{lb}$. \\
Canada \\
\hline
\end{tabular}

\section{LAWN GRASS}

Diamond Brand

40.00

Pearl (Grose for net sacks free) $\quad 30.00$

ORCHARD GRAS3 (Grosa for net sacs free)

Choice Per $100 \mathrm{Lbg}$.

OUR PRIME

PEARL

Prices

" IDAHO “

Mammoth - Domestic Grown

OUR PRIME $\ldots \ldots \ldots \ldots \ldots \ldots \ldots .99 .50$ “ “

Appli-

PEARL....................... “99.00 “ “

LOTUS --10

Medium-Imported-French

About $1 \%$ stained green according to

Federal Law

No.

No. 2

99.50 “ “ “

99.25 “ “ “

NPECIAL_No Buckhorn____ $\quad 98.00$ “ “

TAGS WILL SHOW OUR EXACT PURITY

RED TOP

Extra Fancy Purity $98.20 \quad$ Per 100 Lbs

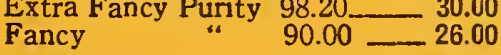

RYE GRASS Der 100 LbE.

Italian 12.50

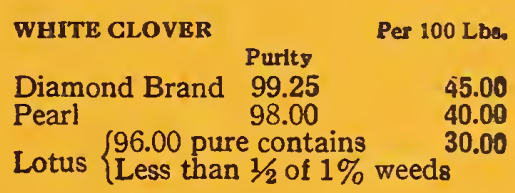

15.50

15.00

14.00

16.00

\section{SWEET GLOVER ALL SCARIFIED}

\section{White Blossom Purity}

Diamond Brand................. 99.75 or better

Per bushel ef 60 pounde

Pearl

99.50 " "

Lotus

98.65 “ "

Yellow Blossom

Diamond Brand.

99.75 “"

Pearl Brand.

99.50 " "

Grundy County (Wh. Blos'm)

Diamond Brand...-..-..-..-. 99.75 “ "

Pearl Brand

TAGS WILL SHOW OUR EXACT PURITY

\section{HUYOGER}

7.80

7.50

Out

7.80

7.50

8.40

Out
No. 1 For Medium, Mammoth, Alsike No. 2 For Alfalfa and Sweet Clover

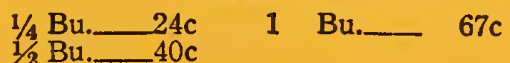
No. 9 For Soy Beans

$1 / 2$ Bu._24c 1 Bu._40c.
Postage Extra

Order by Number and Size

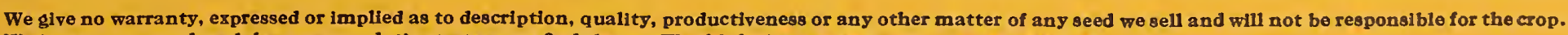
We have an up-to-date laboratory and give tests as we find them. We think they are as near correct as possible.

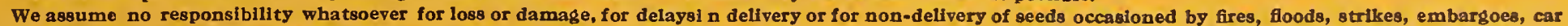
ehortages, or by any other causes beyond our control. 
If you have seed to sell send samples with lowest prices

Before buying take our qualities into consideration

Samples on request

"The Best is the Cheapest"

We sell for future shipment

If interested ask for quotations

\section{Better Seeds \\ Better Crops}

